\title{
Anomalous origin of the left coronary artery from the pulmonary artery diagnosed during transcatheter treatment of severe pulmonary branch stenoses: a potentially life-threatening situation
}

\author{
Laurence Robinson $^{1}$, Alban-Elouen Baruteau ${ }^{2}$, Alain Fraisse ${ }^{1}$ \\ ${ }^{1}$ Royal Brompton and Harefield Hospitals Trust, London, UK; ${ }^{2}$ Evelina London Children's Hospital, London, UK \\ Correspondence to: Alain Fraisse, MD, PhD. Royal Brompton \& Harefield NHS Foundation Trust, Sydney Street, London, SW3 6NP, UK. \\ Email: A.Fraisse@rbht.nhs.uk.
}

\begin{abstract}
We report an 8-year-old female undergoing interventional catheterization for treatment of pulmonary artery stenoses, atrial septal defect and patent ductus arteriosus. Anomalous left coronary artery arising from the pulmonary artery was diagnosed during the procedure. We highlight the importance of recognizing this rare anomaly to avoid the risk of myocardial ischaemia consecutively to abrupt decrease of right ventricular pressure after dilation of pulmonary branches.
\end{abstract}

Keywords: Anomalous origin of the left coronary artery from the pulmonary artery (ALCAPA); pulmonary artery stenosis; coronary steal phenomenon; myocardial ischaemia; transcatheter intervention complications

Submitted Nov 13, 2017. Accepted for publication Feb 01, 2018.

doi: $10.21037 /$ jtd.2018.05.20

View this article at: http://dx.doi.org/10.21037/jtd.2018.05.20

\section{Introduction}

Anomalous origin of the left coronary artery from the pulmonary artery (ALCAPA) is a rarity amongst congenital heart defects, accounting for approximately $0.25-0.5 \%$ of all congenital heart diseases (CHDs) (1,2). Typically, infants with ALCAPA become symptomatic within the first few months of life due to the physiological decrease in pulmonary vascular resistance. This creates a coronary steal phenomenon, which causes cardiac failure secondary to myocardial ischaemia (3). ALCAPA is usually isolated. Rarely, it may present with other congenital heart defects. These include atrial septal defects, ventricular septal defects, patent ductus arteriosus and aortic arch abnormalities, along with more intricate pathologies, such as Tetralogy of Fallot (2). Some of these associated anomalies like unrestrictive ventricular septal defect or patent ductus arteriosus may prevent early diagnosis of the ALCAPA because of persistent increase in pulmonary arterial pressure. Therefore, surgical or interventional management can lead to acute myocardial infarction, cardiac failure and death because of a sudden decrease of pressure in the pulmonary circulation with abrupt coronary steal. We report a unique association of bilateral pulmonary artery stenoses, atrial septal defect and patent ductus arteriosus, associated with an undiagnosed ALCAPA prior to interventional catheterization.

\section{Case presentation}

An 8-year-old female (weight $40 \mathrm{~kg}$, height $145 \mathrm{~cm}$ ) with bilateral pulmonary artery stenoses, secundum atrial septal defect and a patent ductus arteriosus was referred for interventional catheterization. She had been diagnosed in infancy and initially followed in another center where no intervention had been recommended. A diagnostic cardiac catheterization as well as a cardiac CT scan performed there at the age of 6 years did not bring any additional findings. She was asymptomatic. Her cardiac examination revealed a 3/6 ejection systolic murmur radiating to the back. A 12-lead electrocardiogram (ECG) showed normal sinus rhythm with no $\mathrm{Q}$ waves and no evidence of ischemia Echocardiography showed a small secundum atrial septal defect and a moderate patent ductus arteriosus. The proximal pulmonary artery branches were narrowed to 4-mm diameter and severe stenosis was confirmed by continuous Doppler flow with a maximum velocity of $4 \mathrm{~m} / \mathrm{s}$ on each side. Estimated right 

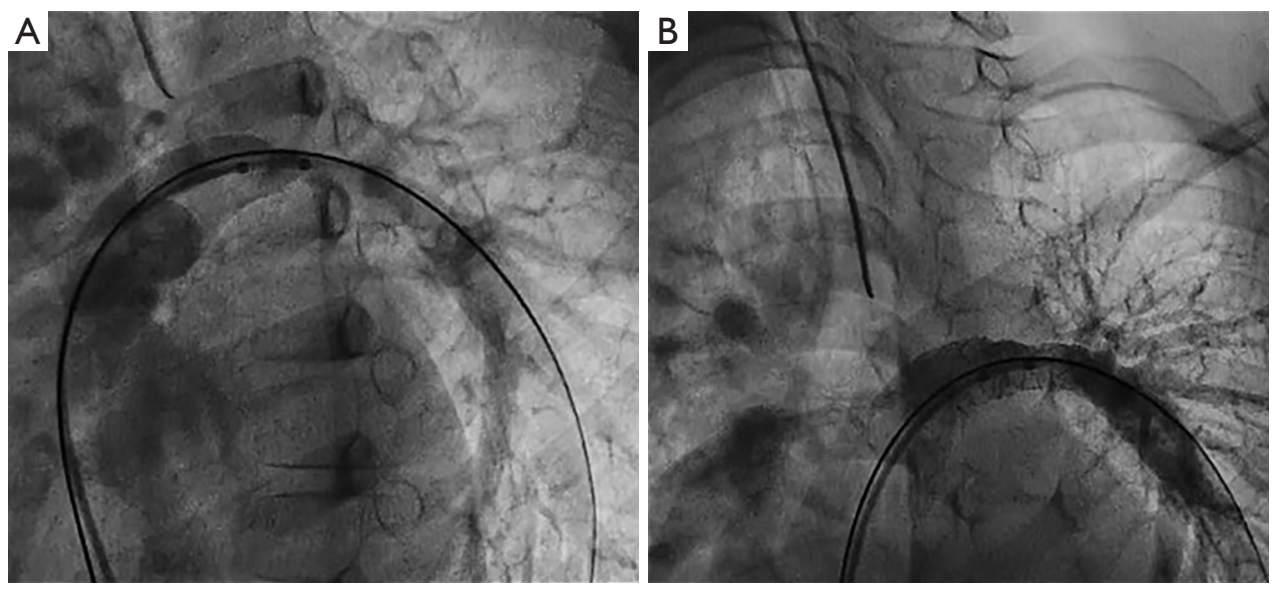

Figure 1 Intervention on the left pulmonary artery. (A) Left pulmonary angiogram in left anterior oblique view showing severe stenosis of the left pulmonary artery; (B) a subsequent left pulmonary angiogram demonstrating an excellent result after stent implantation.

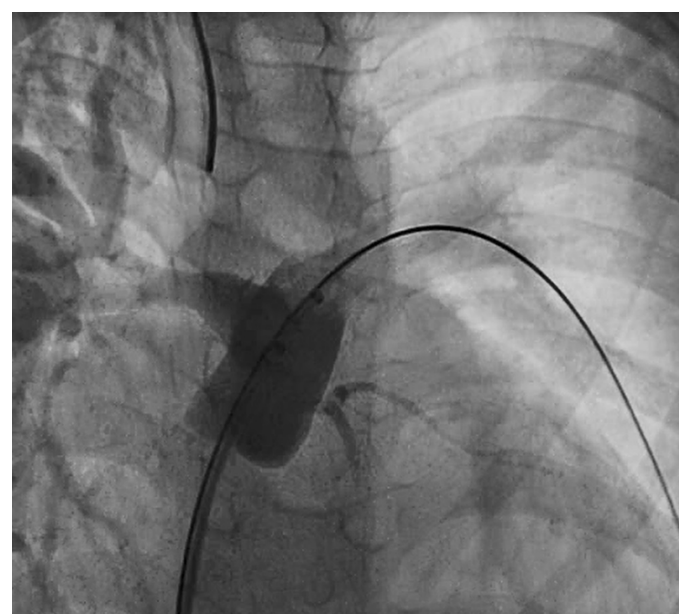

Figure 2 Right ventricular outflow tract angiogram in right anterior oblique view showing a severe stenosis of the right pulmonary artery and an abnormal left coronary artery arising from the pulmonary artery.

ventricular systolic pressure was systemic at $100 \mathrm{mmHg}$. Biventricular systolic function was qualitatively normal. No signs of left ventricular endocardial fibroelastosis were detected, neither was there any degree of mitral valve insufficiency. The patient was discussed in our medicosurgical conference and it was felt that transcatheter intervention was the best option, with the aim to perform pulmonary artery balloon dilation and potentially stent implantation. In addition, both atrial septal defect and patent ductus arteriosus closures were concomitantly planned. Under general anaesthesia and after informed consent, a
4-French sheath was put in the right femoral artery and a 7-French sheath in the right femoral vein. Systemic right ventricular hypertension was confirmed (systolic right ventricular pressure $105 \mathrm{mmHg}$, aorta $107 \mathrm{mmHg}$ ). Left pulmonary artery angiogram showed long and severe stenosis (Figure 1A). Balloon dilation was performed with a $12 \mathrm{~mm} \times 20 \mathrm{~mm}$ Z-Med balloon (NuMED Inc., Hopkinton, NY, USA). This was followed by immediate recoil with no angiographic improvement. A $36 \mathrm{~mm} \times 12 \mathrm{~mm}$ Max LD stent (Medtronic Inc., Minneapolis, MN, USA) was then implanted over a 12-mm BIB balloon (NuMED Inc., Hopkinton, NY, USA) with an excellent result (Figure 1B) and decrease of the systolic right ventricular pressure at $70 \mathrm{mmHg}$ (aorta $102 \mathrm{mmHg}$ ). Before starting intervention on the right pulmonary artery, the initial right ventricle outflow angiogram in right anterior oblique view was reviewed (Figure 2). This demonstrated an ALCAPA. The procedure was immediately abandoned due to the risk of coronary artery steal with a further decrease of the right ventricular pressure. The patient was stable and no ST segment changes were observed on the ECG monitoring. Troponin level remained normal. Surgical reimplantation of the anomalous coronary artery into the aorta was performed 5 days later alongside right pulmonary artery plasty, atrial septal defect and patent ductus arteriosus closure. The post-operative course was uneventful and the patient was discharged home on post-operative day 9. The postoperative echocardiogram confirmed the absence of residual shunt, no residual pulmonary stenosis with normal systolic right ventricular pressure. After 4 years of follow-up the patient remained asymptomatic. No further intervention was needed. 


\section{Discussion}

To our knowledge this is the first case reporting the potential complication of transcatheter intervention due to an undiagnosed ALCAPA. In the present case, the ALCAPA did not result in myocardial ischaemia as the elevated pressure in the pulmonary circulation prevented this. Several cases of unrecognized ALCAPA have been described with surgical repair of associated CHDs $(4,5)$. The difficulty in pre-operative identification of an ALCAPA on a patient's outcome has been highlighted. Laux et al. reported 12 cases of infants with ALCAPA and other CHDs requiring surgical repair. Only 4 (33\%) were diagnosed prior to surgery. The other eight cases were diagnosed either during or after surgery, or at autopsy. Incidental discovery of ALCAPA perioperatively led to compromised post-operative survival (2). Nathan et al. also depicted six cases of ALCAPA with hypoplastic left heart syndrome. Only one case was diagnosed preoperatively. Four of the five cases diagnosed intraoperatively died from cardiac failure and myocardial ischemia (5). Finally, a review of 46 cases of ALCAPA and their associated cardiac defects by Böning et al. did not report any similar association like our case (6).

An undiagnosed ALCAPA may complicate transcatheter intervention the same way this can compromise the result of surgical repair of associated cardiac anomaly. Interestingly in the previous institution where the patient had been initially managed, both a cardiac CT scan and a diagnostic cardiac catheterization had been performed. The ALCAPA was not recognized despite the report mentioning an angiogram in the right ventricle outflow tract. During our procedure, the case was started by a fellow. Although the first angiogram of the right ventricle outflow tract nicely demonstrated the ALCAPA (Figure 2), this was initially missed. Because this first angiogram in right anterior oblique view showed mainly the right pulmonary artery, only the subsequent selective angiogram of the left pulmonary artery was reviewed by the senior interventionist before starting intervention there. Clearly, such intervention on the left pulmonary artery was contraindicated as this could have precipitated potentially fatal myocardial ischemia.

In conclusion, we believe this is the first report of an ALCAPA associated with secundum atrial septal defect, patent ductus arteriosus and bilateral pulmonary artery stenoses. Interventionists must be aware that, although extremely rare, an undiagnosed ALCAPA may be associated with other congenital heart defects, especially when pulmonary artery pressures are high. Our case also highlights the critical importance to carefully review and analyze any angiogram before starting any intervention.

\section{Acknowledgements}

None.

\section{Footnote}

Conflicts of Interest: The authors have no conflicts of interest to declare.

Informed Consent: Informed consent was obtained from the patient for publication of this manuscript and any accompanying images.

\section{References}

1. Dodge-Khatami A, Mavroudis C, Backer CL. Anomalous origin of the left coronary artery from the pulmonary artery: collective review of surgical therapy. Ann Thorac Surg 2002;74:946-55.

2. Laux D, Bertail C, Bajolle F, et al. Anomalous left coronary artery connected to the pulmonary artery associated with other cardiac defects: a difficult joint diagnosis. Pediatr Cardiol 2014;35:1198-205.

3. Schneider T, Rickli H, Gliech V, et al. Bland-White-Garland syndrome and atrial septal defect - rare association and diagnostic challenge. Clin Res Cardiol 2006;95:295-300.

4. Bobylev D, Meschenmoser L, Boethig D, et al. Surgical repair of Shone's complex with anomalous origin of the left coronary artery arising from the right pulmonary artery. Interact Cardiovasc Thorac Surg 2015;20:439-42.

5. Nathan M, Emani S, Marx G, et al. Anomalous left coronary artery arising from the pulmonary artery in hypoplastic left hearts: case series and review of literature. J Thorac Cardiovasc Surg 2011;142:225-7.

6. Böning U, Sauer U, Mocellin R, et al. Anomalous coronary drainage from the pulmonary artery with associated heart and vascular abnormalities. Report on 3 patients and review of the literature. Herz 1983;8:93-104.

Cite this article as: Robinson L, Baruteau AE, Fraisse A. Anomalous origin of the left coronary artery from the pulmonary artery diagnosed during transcatheter treatment of severe pulmonary branch stenoses: a potentially life-threatening situation. J Thorac Dis 2018;10(5):E344-E346. doi: 10.21037/ jtd.2018.05.20 